\title{
Results From Ireland North and South's 2016 Report Card on Physical Activity for Children and Youth
}

\author{
Deirdre M. Harrington, Marie Murphy, Angela Carlin, Tara Coppinger, Alan Donnelly, Kieran P. Dowd, \\ Teresa Keating, Niamh Murphy, Elaine Murtagh, Wesley O'Brien, Catherine Woods, and Sarahjane Belton
}

\begin{abstract}
Background: Physical activity (PA) is a key performance indicator for policy documents in both the Republic of Ireland and Northern Ireland. Building on baseline grades set in 2014, Ireland's second Report Card on Physical Activity for Children and Youth allows for continued surveillance of indicators related to PA in children and youth. Methods: Data and information were extracted and collated for 10 indicators and graded using an international standardized grading system. Results: Overall, 7 grades stayed the same, 2 increased, and 1 decreased. Grades were assigned as follows: Overall PA, $D$ (an increase); Sedentary Behavior (TV), C-; Physical Education, D-; Active Play, Incomplete/Inconclusive (INC); Active Transportation, D; School, D (a decrease); Home (Family), INC; Community and the Built Environment, B+ (an increase); and Government, INC. Unlike 2014's report card, different grades for the Republic $(C$-) and Northern Ireland $(C+)$ were assigned for Organized Sport Participation. Conclusions: Although the grade for Overall PA levels increased to a $D$, this may reflect the increased quality and quantity of data available. The double burden of low PA and high sedentary levels are concerning and underscore the need for advocacy toward, and surveillance of, progress in achieving targets set by the new National Physical Activity Plan in the Republic and obesity and sport plans in the North.
\end{abstract}

Keywords: sedentary, sport, school, transport, play

Physical activity (PA) guidelines in Ireland recommend that children should accumulate at least 60 minutes of moderate-tovigorous PA (MVPA) daily for health benefits. ${ }^{1,2}$ Despite this global recommendation, and the widely reported benefits for participation in PA, in 2014 we reported that only $25 \%$ of children on the island of Ireland were meeting PA recommendations. ${ }^{3}$ In the Republic of Ireland's recently released National Physical Activity Plan, ${ }^{4}$ there is a focus on children and young people, with a specific target that "children and young people learn the necessary skills for confident engagement with physical activity and will have opportunities to adopt an active way of life." In Northern Ireland, the Fitter Futures for All Framework ${ }^{5}$ includes the long-term outcomes of "a greater proportion of children and young people are achieving recommended levels of physical activity" in the prevention and management of obesity.

In 2014, Ireland (in a cross-border effort involving both the Republic of Ireland and Northern Ireland) $)^{3,6}$ joined 14 other countries in launching their Report $\mathrm{Card}^{7}$ which acted as a baseline for surveillance of PA promotion efforts and to become an advocacy tool to push for changes in PA policy and programming. The 2014

Harrington is with the University of Leicester, Leicester, UK. M Murphy is with Ulster University, Co. Antrim, N. Ireland. Carlin, Woods, and Belton are with Dublin City University, Dublin, Ireland; Carlin is also with the University of Limerick, Limerick, Ireland. Coppinger is with the Cork Institute of Technology, Cork, Ireland. Donnelly is with the University of Limerick, Limerick, Ireland. Dowd is with the Athlone Institute of Technology, Athlone, Ireland. Keating is with the Institute of Public Health in Ireland, Dublin, Ireland. N Murphy is with the Waterford Institute of Technology, Waterford, Ireland. Murtagh is with Mary Immaculate College, Limerick, Ireland. O'Brien is with University College Cork, Cork, Ireland. Harrington (dh204@le.ac.uk) is corresponding author. edition was well received by media and by stakeholders and, to continue the momentum, the 2016 Ireland North and South Report Card has been produced by Active Healthy Kids Ireland as part of the Active Healthy Kids Global Alliance. Although the Republic of Ireland and Northern Ireland are 2 independent nations on the same island (the former is an independent autonomous country whereas the latter is part of the sovereign state of the United Kingdom of Great Britain and Northern Ireland) under the 1998 Good Friday Agreement a power-sharing executive was set up in Northern Ireland with devolved powers over areas of legislation and policy relating to areas which impact on children's physical activity (transport, education, for example). Given attempts by both governments to encourage cross-border co-operation between the 2 jurisdictions our intention was to produce a unified report for the whole island of Ireland, North and South combined. Where the evidence for a given indicator differed between the Republic and Northern Ireland we agreed to assign different grades but to present these within a single all-island Report Card. The aim of the present paper is to summarize the results of the 2016 Ireland North and South Report Card.

\section{Methods}

The development and production of Ireland's 2016 Report Card was undertaken by the research work group (RWG) comprised of 12 PA and health researchers from Universities and public bodies. The RWG was chaired by Harrington and the work was led by 2 principal investigators (PIs); one based in Northern Ireland (M. Murphy) and the other based in the Republic of Ireland (Belton). A part-time research assistant (Carlin) identified, extracted, and collated any new data and information from academic articles, datasets and policy documents between 2011 and 2015 following international guidance ${ }^{8}$ and methods used in 2014. Sources were 
identified through database and internet searches, contact with the RWG, and contact with other research groups and stakeholders across Ireland. Data were presented, discussed and graded by wider RWG at a meeting in March 2016. Factors considered when grading were sample size, methodology, whether there was disparities or inequities in the data (for example, boys more active than girls) and how well the most recent data matched with the benchmark set for that indicator. The benchmark was set as the target that children themselves should achieve to promote health or that the settings should have in place to support PA. Grades from $A$ to $F$ (including "+" or "-") could be assigned with an Incomplete ("inconclusive") grade being available if not enough data exist on that indicator. As a range of grades were proposed for each indicator (ie, raise, lower, or no change from 2014), the data were further scrutinized by the PIs and RWG chair. A draft set of grades and rationale was circulated to the RWG and each member provided further feedback. Draft grades and accompanying rationale were then presented to stakeholders from 14 agencies in April 2016. Stakeholders inputted on the relevance of the grades to their agency's work and provided policy and 'real world' context and a voice for end users 'on the ground' on how to frame the messaging within the Report Card to ensure maximum relevance and impact. Stakeholders included those representing health and wellbeing, health promotion, primary and secondary physical education (PE), early childhood, play, transport, education, sport, and disability. Following this meeting the RA, the PIs and the chair set about preparing the Report Card, consulting further with the stakeholders and other RWG members as required.

The 10 indicators included in Ireland's 2016 Report Card are consistent with the 2014 edition: Overall Physical Activity; Sedentary Behavior (TV viewing); Organized Sport Participation; Physical Education (PE); Active Play; Active Transportation; School; Community and the Built Environment; Home (Family); and Government. The following datasets formed the basis of the 2016 grades but are supplemented, when mentioned, by other local studies or gray literature:

\section{Republic of Ireland Datasets}

Growing Up in Ireland (GUI) Infant and Child Cohorts. ${ }^{9,10}$ GUI is a longitudinal study of 2 representative cohorts of children in the Republic of Ireland funded by the Department of Children and Youth Affairs and undertaken by a consortium of researchers led by the Economic and Social Research Institute and Trinity College Dublin. Data from Wave 3 of the infant cohort, followed up at age 5 years $(n=\sim 9000$ children and their care-givers; collected in 2013), and Wave 2 of the child cohort, followed up at age 13 years $(n=$ 7400; data collected August 2011 to March 2012) are reported.

Health Behavior of School-Aged Children (HBSC). ${ }^{11,12}$ HBSC is a cross-national school-based survey of children and data have been collected in the Republic of Ireland over 5 waves (1997-2014) by the National University of Ireland Galway and funded by the World Health Organization and the Department of Health. We report data from the 2013-2014 $(n=13,611)$ waves collected on a representative sample of 11- to 15-year-olds.

Children's Sport Participation and Physical Activity (CSPPA Plus). ${ }^{13,14}$ The CSPPA study collected data on 5397 children and youth aged 10 to 18 years in 2009 in the Republic of Ireland, with the aim of providing a national database on PA, PE, and sport participation in youth. ${ }^{15}$ The CSPPA Plus study involved following up this cohort of youth 5 years after initial contact and reported data on 873 participants (response rate $=22.3 \%$, aged 15 to 21 years).

\section{Northern Ireland Datasets}

Young Persons' Behavior and Attitudes Survey (YPBAS). ${ }^{16,17}$ YPBAS is commissioned by Northern Ireland government departments, and designed and administered by the Central Survey Unit to query topics relevant to the lives of young people. The fourth round of the survey was administered to 7076 pupils with schools randomly assigned to complete 1 out of 2 versions of the questionnaire. We report on data from Version A of the questionnaire, collected from 3174 11- to 16-year-olds in 2013.

UK Millennium Cohort Study Wave 5 (MCS5). ${ }^{18}$ This is a longitudinal study following the lives of children born in the UK. It is funded by the Economic and Social Research Council and run by the Centre for Longitudinal Studies, UK. Wave 5 data were collected in 2012 when children were aged 11 years $(n=13,287)$. The survey included interviews with parents and a self-completion questionnaire for the child participant with data for Northern Ireland specifically reported herein $(\mathrm{n}=1931)$.

Northern Ireland Travel Survey. ${ }^{19}$ The Northern Ireland Continuous Household Survey is administered by Central Survey Unit and the Northern Ireland Statistics and Research Agency. Since 2013, the survey has included parent report of method of travel to/from school. We report data on the 2014-2015 survey on 623 primary school and 525 postprimary school children from Northern Ireland.

\section{Results}

The 2016 Ireland North and South Report Card is the second biennial assessment of indicators related to PA of children and youth living on the island of Ireland. The grades are included in Table 1, while the main document cover can be seen in Figure 1.

Table 1 Grades According to Physical Activity Indicator in the 2016 Ireland North and South Report Card on Physical Activity for Children and Youth

\begin{tabular}{lc}
\hline Indicator & Grades \\
\hline Overall Physical Activity Levels & $D$ \\
Organized Sport Participation & $C$ - RoI / C+ NI \\
Physical Education & $D-$ \\
Active Play & $I N C$ \\
Active Transportation & $D$ \\
Sedentary Behaviors & $C$ - \\
Home (family) & $I N C$ \\
School & $D$ \\
Community and the Built Environment & $B+$ \\
Government strategies and investments & $I N C$ \\
\hline
\end{tabular}

Note. The grade for each indicator is based on the percentage of children and youth meeting a defined benchmark: $A$ is $81 \%$ to $100 \%$; $B$ is $61 \%$ to $80 \%$; $C$ is $41 \%$ to $60 \%, D$ is $21 \%$ to $40 \% ; F$ is $0 \%$ to $20 \%$; INC is Inconclusive/incomplete data. Abbreviations: RoI, Republic of Ireland; NI, Northern Ireland. 


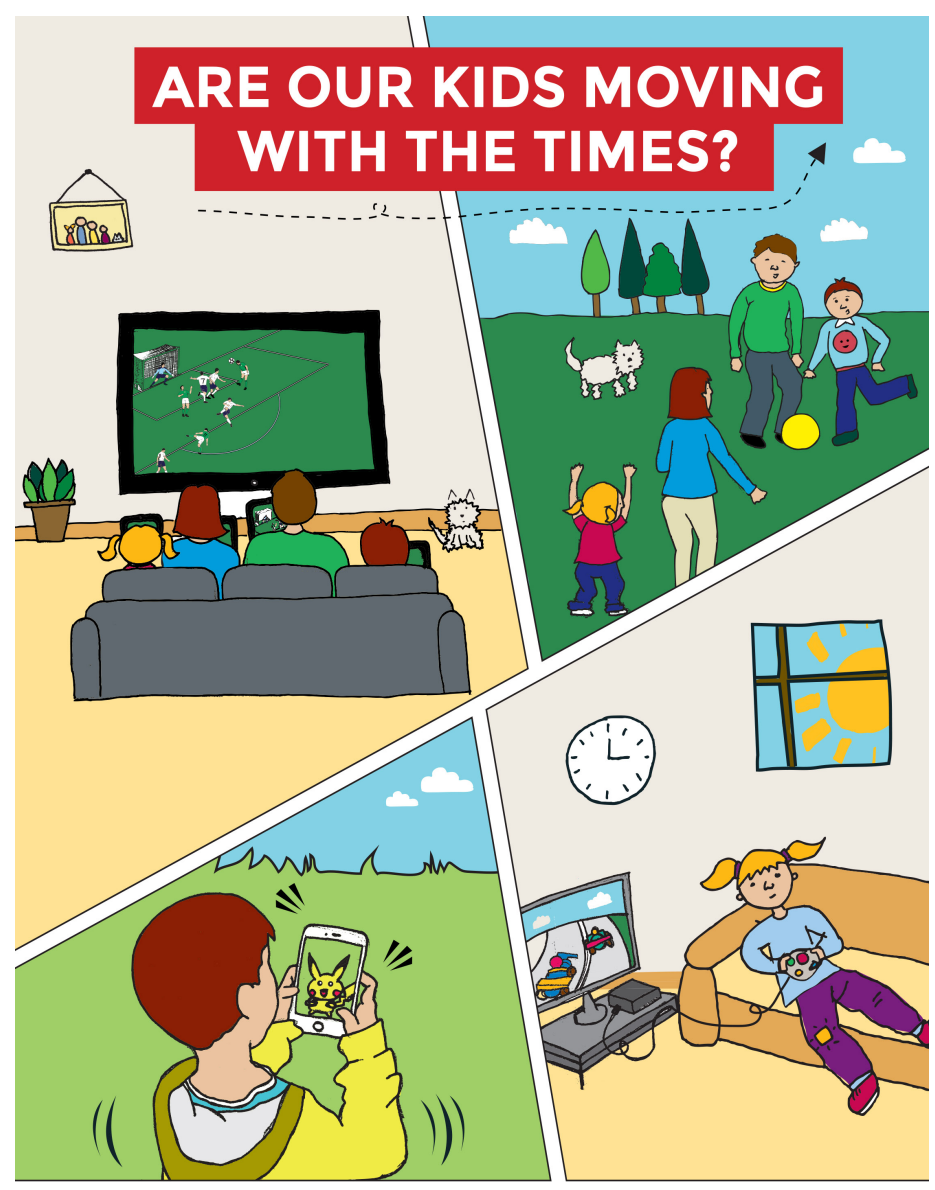

The 2016 Ireland North and South Report Card on Physical Activity for Children and Youth

Figure 1 - Front cover of the 2016 Ireland North and South Physical Activity Report Card.

\section{Discussion}

Overall, 1 indicator decreased (School), 2 indicators (Overall PA Levels and Community and the Built Environment) increased, and Sport was graded separately for The Republic and Northern Ireland. The strengths and limitations to the data available for each indicator are discussed in the long form of the 2016 Report Card..$^{20}$ Although the RWG are encouraged by the quantity of new data, and the quality of the methods, we found that data between waves of studies were not always consistent. This made it difficult at times to compare with the 2014 edition and the benchmarks set for this international Report Card process.

\section{Overall Physical Activity: D}

Using the criterion of percentage of children meeting the PA guideline of at least 60 minutes of MVPA every day, ${ }^{1,2}$ this indicator was graded $D$. This is an increase from the $D$ - awarded in the 2014
Report Card. Five large datasets representing differing age groups from the Republic and Northern Ireland were considered. Based on self-report methods $14 \%$ to $41 \%$ of children meet the PA benchmark. From the Republic of Ireland 23\% of 10- to 17-year-olds ${ }^{11,12}$ met the benchmark while in Northern Ireland $41 \%$ of 5- to 18 -year-olds, ${ }^{19}$ $24 \%$ of 9 - to 11 -year-olds ${ }^{21}$ and $14 \%$ of 11 - to 16 -year-olds. ${ }^{17}$ met the benchmark. Although data from a longitudinal study in the Republic found that $25 \%$ of 9 -year-olds met the benchmark ${ }^{22}$ (as reported in the 2014 Report Card), this question was not asked again when the children were followed-up at 13 years of age in wave $2 .{ }^{9}$ Both sex and age differences were evident as for boys and girls, respectively, $31 \%$ and $45 \%$ at age $11,16 \%$ and $36 \%$ at age 13 , and $9 \%$ and $25 \%$ at age 15 met the benchmark. ${ }^{12}$ Using objective measures of PA from regional samples showed that $22 \%$ of 8 - to 11-year-olds (unpublished from ${ }^{23}$ ) and $32 \%$ of 11 - to 14 -year-olds ${ }^{24}$ met the benchmark.

Though not applicable to all indicators, a weighted mean for Overall PA, from self-reported and objective methods, found that $49 \%$ and $32 \%$, respectively, met the benchmark indicating a $D$ grade overall. Though this increase can be heralded as a positive movement, this increase may reflect the greater quantity and improved quality of measurement studies that have been carried out and published between 2010 and 2015

\section{Organized Sport Participation: C- / C+}

Using the target of percentage of children participating in sport twice per week, ${ }^{25}$ this indicator was graded differently for the Republic and Northern Ireland. Due to a lack of new data that matched the benchmark, a $C$ - was awarded in the Republic of Ireland, indicating no change from the 2014 Report Card. Northern Ireland was awarded a $C+$, indicating a slight increase from 2014. In Northern Ireland, $65 \%$ of young people aged 11 to 16 reported participation in $2+$ hours of sport/PA outside of school each week. ${ }^{17}$ Some $54 \%$ of parents of 11-year-olds in Northern Ireland reported that their child goes to a club or class to do sport/other PA $\geq 2$ days per week. ${ }^{18}$ This compares to $40 \%$ of children meeting the benchmark from the same study in the 2014 Report Card. In the Republic, recent datasets ${ }^{9-12}$ have not differentiated between school sports club participation (which would be included in the school indicator) and participation in extraschool clubs. Inequities are evident with girls, those from lower social classes, and older children less likely to participate regularly in sports clubs. ${ }^{11,12,17}$

\section{Active Play: INC}

Although active play is a contributor to MVPA recommendations, ${ }^{1}$ there continues to be no universally agreed benchmark to allow for this indicator to be assessed independently from other PA, although "\% of children and youth engaging in unstructured/unorganized active play for several hours a day" has been proposed. ${ }^{26}$ Although 2 large datasets include data on active play, the mismatch between the arbitrary benchmark and the questions asked means this grade remains as INC in 2016. Analysis of data from the Republic ${ }^{10}$ shows that an average of $42 \%$ of 5 -year-olds undertake some form of active play every day, which would suggest a $C$ - for the Republic, while $72 \%$ of children in Northern Ireland reported playing sports or active games inside or outside but not at school on most days of the week, ${ }^{18}$ which would suggest a $B$ grade; although that value includes 'outdoor sports' so is likely to be somewhat inflated. 


\section{Active Transportation: $D$}

Using the percentage of children reporting active transport to or from school each day, this indicator was graded $D$, which represents no change from the 2014 Report Card. Data from larger studies (>2000 participants) ${ }^{16,27-29}$ suggest we are succeeding with $20 \%$ to $29 \%$. Parent reported data from Northern Ireland showed $51 \%$ of children usually (rather than each day) walked or cycled to school. ${ }^{11}$ Inequalities in active transportation are still evident, with children from rural areas less likely to active commute than their urban counterparts. ${ }^{19}$ Sex gaps evident for other indicators may not be as obvious for active transport-for example, $23 \%$ of males and $25 \%$ of females use of active transport in a local sample of 287712 - to 20 -year-olds in the Republic. ${ }^{28}$

\section{Sedentary Behavior (TV Viewing): C-}

Based on the benchmark of percentage of children watching $<2$ hours of TV/day, ${ }^{30}$ this indicator was graded as $C$-, representing no change from 2014. Data from the Republic indicated that $~ 53 \%$ of 13 -year-olds watched $<2$ hours TV/videos/DVDs each day. ${ }^{9}$ Using a question that captures total screen time, $58 \%$ of 5 -year-olds spend $\leq 2$ hours engaged in screen time of any type on an average weekday. ${ }^{10}$ From HBSC 2014, 50\% of 11- to 15-year-olds reported watching $<2$ hours TV/day, ${ }^{11,12}$ compared with $46 \%$ from $2009-2010$ reported in Ireland's 2014 Report Card. Data also indicates that TV viewing increases with age as percentage of youths meeting the benchmark decreased by $11 \%$ between ages 11 and 15 years. ${ }^{11,12}$ Data from Northern Ireland shows that $60 \%$ of 11 -year-old children spent $<2$ hours watching programs or films on any screen on the average school day. ${ }^{18}$

\section{Physical Education (PE): D-}

Based on the benchmark of the discrepancy between the recommended time ( $\geq 1$ hour/week for primary ${ }^{31}$ and 2 hours/week for postprimary ${ }^{32}$ in the Republic and 2 hours/week in Northern Ireland $)^{33}$ allocated to PE in schools, and the amount actually reported by students or schools, this indicator was graded as a $D$-, representing no change from the last report card. Since the 2014 Report Card, there has been a lack of published data reporting the prevalence of children receiving the recommended time for weekly $\mathrm{PE}$ and data from Northern Ireland will include time in school games lessons (PE classes where students take part in a range of sports activities) as well as PE. $69 \%$ of 11- to 16-year-olds reported being involved in $\mathrm{PE} /$ games lessons for $\geq 2$ hours/week. ${ }^{17}$ Age-related differences were apparent, with $79 \%$ of those aged 12 and under reporting $\geq 2$ hours/ week, compared with $57 \%$ of those aged $\geq 16 .{ }^{17}$ Data has shown that the time allocated to PE varies between year groups within and across the school settings in the Republic ${ }^{34}$ and Northern Ireland. ${ }^{35}$

\section{Home (Family): INC}

No accepted standard benchmark for this indicator currently exists either internationally or in Ireland North or South. The grade remains $I N C$ due to a lack of an established benchmark. Reviews of the supports for children's PA highlight some elements of the home social environment that could act as a benchmark such as family social support. ${ }^{36,37}$ Available data from the Republic of Ireland show that $22 \%$ of parents reported undertaking sport or PA with their 5-yearold children everyday ${ }^{10}$ while in Northern Ireland this value was $3 \%$ for parents who play sports or physically active games outdoors or indoors every day/almost every day. ${ }^{18}$

\section{School: $D$}

Using the benchmark of the percentage of children participating in $2+\mathrm{hr} /$ week of extracurricular sport and school based recreation, ${ }^{38}$ this indicator was graded $D$, which indicates a drop from 2014 ( $C$-). In Northern Ireland, $27 \%$ of 11- to 16-year-olds normally stayed behind after school to take part in sport or PA for $2+$ hours/week $^{17}$ compared with $49 \%$ reported in the 2014 report card. However, the way the more recent question was asked is better suited to the benchmark, as it focuses instead on staying behind at school for sport and PA whereas data used in the 2014 report card encompassed PE, games, and playing for a school team. The observed difference in results between the 2 waves is likely due to the change in question asked as opposed to a change in frequency of participation. Data from the Republic previously included in the 2014 Card found that $42 \%$ of primary and $57 \%$ of postprimary students reported participating in extracurricular sport at least twice/week. This compared with. $30 \%$ reporting the same participation in 2014 when the children were older ( $>15 \mathrm{yrs}) \cdot{ }^{13,14}$

\section{Community and the Built Environment: $B+$}

Using the benchmark of the percentage of parents or adolescents perceiving their local area and PA facilities as safe or good quality, this indicator was graded $B+$, representing an increase from the 2014 grade $(B)$. In the Republic, $94 \%$ of parents strongly agreed/agreed their local area was safe for their 13-year-old child. ${ }^{9}$ In Northern Ireland, $94 \%$ of 11- to 16-year-olds reported feeling safe in the area they live, while $64 \%$ felt the play/leisure facilities in their area were good. ${ }^{16} \mathrm{~A}$ further $89 \%$ of 11 -year-olds reported that the area they live in was very safe/safe to walk, play, or hang out in during the day. ${ }^{18}$ A study including children from Ireland North and South reported that $94 \%$ of 7 - to 15 -year-olds believed their neighborhood to be safe/very safe. ${ }^{39}$

\section{Government: INC}

Although difficult to obtain or nonexistent in many jurisdictions, ${ }^{40}$ quantification of the investment into PA and the implementation of PA policy would be the ideal benchmark that all jurisdictions would aim to capture. Both the Republic of Ireland and Northern Ireland include the promotion of children's PA in policy and strategy documents that span several sectors, including sport, education, health, and transport. Since the 2014 Report Card there has been some progress from the government and public sector, most notably the publication of the National Physical Activity Plan in the Republic of Ireland. ${ }^{4}$ This plan sets out 60 actions-14 of which are specifically designed to increase PA in children and young people. Each action identifies a lead and partner agencies and timescales for the delivery. ${ }^{4}$ In Northern Ireland there is a notable absence of a bespoke national PA policy or plan, however objectives to increase children's participation in PA and sport are embedded within the strategic plans and policy objectives of a number of other organizations and policy objectives including the Fit Futures Obesity Action Plan 2012-20225,41 and Sport Matters: the Northern Ireland Strategy for Sport \& Physical Recreation 2009-2019. ${ }^{38}$ The unavailability of data on investment and the extent of the workforce devoted to implementing policy related to children's PA, and the lack of clear mechanisms for tracking children's PA targets means that much of the key evidence required on the Government indicator is lacking. However, the RWG acknowledge the progress made in policy in the Republic, and the cross-sector working in both jurisdictions. 


\section{Strengths and Limitations}

The RWG included representatives from across the island of Ireland and ensures cross-border working in the area of PA advocacy. More agencies participated in the stakeholder consultation process in 2016 than in the 2014 edition, which helps ensure that the views of the end users of this advocacy document are heard. Although grades are based on the best available data up to 2015, there were significant research gaps. Firstly, data collected in large scale studies did not always match the benchmark set in the international Report Card process (for example PE and overall PA indicators). Rather than the Report Card benchmarks changing to fit the available data, we suggest that future studies ask questions that align with Report Card benchmarks included herein as they follow national and international PA recommendations and are being used as global indicators of physical activity. Secondly, there are gaps in data available for certain indicators (eg, active play), or groups such as early childhood in general and underrepresented children (eg, disabilities, minority children, and those in care).

\section{Conclusion}

The grade for Overall PA has increased from the 2014 edition of Ireland's Report Card, which may also reflect an improvement in the quantity and quality of data available. Yet this grade is still only a $D$, which means there is much room for improvement as more than half of children on the island of Ireland are still not meeting the recommended levels to obtain the myriad of physical, mental, and social benefits that being active brings. The RWG are encouraged by the release of the Republic of Ireland's National Physical Activity Plan as this is the Republic's first clear 'stand-alone' single issue PA policy statement which has the potential to make a substantial contribution to a change in population levels of PA. Equally, the embedding of PA objectives in a number of Northern Ireland strategic plans and related agendas to achieve synergist policy impacts is welcomed. However, the RWG believe that to see change in the health and wellbeing of Ireland's current children and future generations urgent, measurable action is necessary. This will require continued advocacy to policy makers, practitioners, and parents to ensure PA remains a key priority.

\section{Acknowledgments}

The authors thank the following individuals for their contributions to Ireland's Ireland Report Card 2016: Dr. Mark Tremblay, Joel Barnes, Dr Peter Katzmarzyk and the wider Active Healthy Kids Global Alliance team members. We also thank Dr Martin Dempster (Queen's University Belfast) for assistance with data analysis. We thank the stakeholders who provided their valuable opinion and insight into the grades. This work was supported by grants from the Public Health Agency (NI), Sport Northern Ireland, the Irish Heart Foundation, and the Institute of Public Health in Ireland. The views expressed herein are the authors and do not necessarily represent the views of the funders or the affiliated institutions.

\section{References}

1. Department of Health and Children. Get Ireland active! The national guidelines on physical activity for Ireland. Dublin: Health Service Executive; 2009. Available from http://health.gov.ie/wp-content/ uploads/2014/03/active_guidelines.pdf. Accessed March 2, 2016.

2. UK Chief Medical Officers. Start active, stay active: A report on physical activity for health from the four home countries' chief medical officers. Department of Health; 2011. Available from www.dh.gov. uk/en/Publicationsandstatistics/Publications/PublicationsPolicyAndGuidance/DH_128209. Accessed March 2, 2016.

3. Harrington DM, Belton S, Coppinger T, et al. Ireland's 2014 Report Card on Physical Activity in Children and Youth. 2014. Available from www.dcu.ie/sites/default/files/shhp/docs/ReportCardIreland2014_ LongForm_Final.pdf doi:10.1123/jpah.2014-0166. Accessed January 27, 2016.

4. Department of Health and the Department of Transport TaS. Get Ireland active! National Physical Activity Plan for Ireland. Dublin; 2016. Available from http://www.getirelandactive.ie/Professionals/ National-PA-Plan.pdf. Accessed February 4, 2016.

5. Department of Health Social Services and Public Safety. A fitter future for all: Framework for preventing and addressing overweight and obesity in Northern Ireland 2012-2022. 2012. Available from http://www. northerntrust.hscni.net/pdf/FitfuturesforallObesityFrameworkNI2012. pdf. Accessed February 4, 2016.

6. Harrington DM, Belton S, Coppinger T, et al. Results From Ireland's 2014 Report Card on Physical Activity in Children and Youth. $J$ Phys Act Health. 2014;11(Suppl 1):S63-S68. PubMed doi:10.1123/ jpah.2014-0166

7. Tremblay MS, Gray CE, Akinroye KK, et al. Physical activity of children: a global matrix of grades comparing 15 countries. J Phys Act Health. 2014;11(Supp 1):S113-S125. PubMed doi:10.1123/ jpah.2014-0177

8. Colley RC, Brownrigg M, Tremblay MS. A model of knowledge translation in health: The Active Healthy Kids Canada Report Card on Physical Activity for Children and Youth. Health Promot Pract. 2012;13(3):320-330. PubMed

9. Economic and Social Research Institute Trinity College Dublin and the Office of the Minister for Children and Youth Affairs. Growing Up in Ireland Child Cohort Wave 2. 2013.

10. Economic and Social Research Institute Trinity College Dublin and the Office of the Minister for Children and Youth Affairs. Growing Up in Ireland Infant Cohort Wave 3. 2013.

11. Gavin A, Keane E, Callaghan M, Molcho M, Kelly C, Nic Gabhainn S. The Irish Health Behaviour in School-aged Children (HBSC) Study 2014. Galway, Ireland: Health Promotion Research Centre at the National University of Ireland. Galway: NUIG; 2015.

12. Inchley J, Currie D. Growing up unequal: gender and socioeconomic differences in young people's health and well-being. Health Behaviour in School-aged Children (HBSC) study: International report from the 2013/2014 survey. World Health Organization; 2016. Available from http://www.euro.who.int/en/publications/abstracts/growingup-unequal-gender-and-socioeconomic-differences-in-youngpeoples-health-and-well-being.-health-behaviour-in-school-agedchildren-hbsc-study-international-report-from-the-20132014-survey. Accessed April 5, 2016.

13. Hardie Murphy M, Rowe DA, Woods CB. Sports participation in youth as a predictor of physical activity: a 5-year longitudinal study. J Phys Act Health. 2016;13(7):704-11. PubMed

14. Hardie Murphy M, Rowe DA, Woods CB. Impact of physical activity domains on subsequent physical activity in youth: a 5-year longitudinal study. J Sport Sci. 2016:1-7.

15. Woods CB, Tannehill D, Quinlan A, Moyna N, Walsh J. The Children's Sport Participation and Physical Activity Study (CSPPA). Dublin, Ireland: School of Health and Human Performance, Dublin City University and the Irish Sports Council; 2010. Available from https:// www.ucd.ie/t4cms/CCLSP_Study_Report1.pdf. Accessed January 27, 2016.

16. Northern Ireland Statistics Research Agency. Young persons' behaviour and attitudes survey topline headline bulletin. NISRA Belfast; 
2013. Available from http://www.csu.nisra.gov.uk/survey.asp96.htm. Accessed January 27, 2016.

17. Department of Culture Arts and Leisure. Experience of sport and physical activity by young people in Northern Ireland: Findings from the Young Persons' Behaviours and Attitudes Survey 2013. Northern Ireland Research and Statistics Agency and Department of Culture, Arts and Leisure; 2014. Available from https://www.communities-ni. gov.uk/publications/experience-sport-and-physical-activity-by-youngpeople-northern-ireland-2013. Accessed January 28, 2016.

18. University of London, UCL Institute of Education, Centre for Longitudinal Studies. Millennium Cohort Study: Fifth Survey. 6th ed. Colchester, Essex: UK Data Archive; 2012.

19. Department for Regional Development. Agency NISaR. Method of Travel to/from School by Pupils in NI, 2014/2015. Department for Regional Development and 2015. Available from https://www.gov. uk/government/statistics/method-of-travel-tofrom-school-by-pupilsin-ni-20132014. Accessed February 12, 2016.

20. Active Healthy Kids Ireland. Ireland's 2016 Report Card on Physical Activity in Children and Youth. 2016. Available from www.activehealthykidsireland.eu.

21. Breslin G, Gossrau-Breen D, McCay N, Gilmore G, MacDonald L, Hanna D. Physical activity, gender, weight status, and wellbeing in 9-to 11-year-old children: a cross-sectional survey. J Phys Act Health. 2012;9(3):394. PubMed doi:10.1123/jpah.9.3.394

22. Economic Social and Research Institute, Trinity College Dublin, Office of the Minister for Children and Youth Affairs. Growing up in Ireland Report 1. Dublin 2009. Available from http://www.growingup. ie/fileadmin/user_upload/documents/1st_Report/Barcode_Growing_Up_in_Ireland_-_The_Lives_of_9-Year-Olds_Main_Report.pdf. Accessed January 28, 2016.

23. Keane E, Kearney PM, Perry IJ, Browne GM, Harrington JM. Diet, physical activity, lifestyle behaviors, and prevalence of childhood obesity in Irish children: The Cork Children's Lifestyle Study Protocol. JMIR Res Protoc. 2014;3(3):e44. PubMed doi:10.2196/resprot.3140

24. Belton S, O'Brien W, Issartel J, McGrane B, Powell D. Where does the time go? Patterns of physical activity in adolescent youth. J Sci Med Sport. 2016; in press. PubMed doi:10.1016/j.jsams.2016.01.008

25. LISPA Working Group. Lifelong involvement in sport and physical activity: The LISPA Model. Available from http://www.sportireland.ie/ Coaching-Ireland/Life-Long-Involvement-In-Sport-Physical-Activity/ LISPA.pdf. Accessed October 31, 2016.

26. Particip ACTION. The 2015 ParticipACTION Report Card on Physical Activity for Children and Youth. 2015. Available from https://www. participaction.com/sites/default/files/downloads/Participaction2015ReportCard-FullReport_5.pdf. Accessed March 10, 2016.

27. Sullivan L, Nic Gabhainn S. Physical activity, active travel and exercise among school children in Ireland 2010. HBSC Ireland. Galway, Ireland: Health Promotion Research Centre at the National University of Ireland, NUI Galway; 2012. Available from http://www.nuigalway.ie/ hbsc/documents/2012_sr_1s_physical_activity_active_travel_exercise.pdf. Accessed February 18, 2016.

28. Delaney P. Sport and Recreation Participation and Lifestyle Behaviours in Waterford City Adolescents. Dublin, Ireland: Waterford Institute of Technology And The Irish Sports Council; 2013. Available from https://www.irishsportscouncil.ie/Research/Adolescent_Lifestyle_2013_/Adolescent.pdf. Accessed March 2, 2016.
29. Murtagh EM, Dempster M, Murphy MH. Determinants of uptake and maintenance of active commuting to school. Health Place. 2016;40(July):9-14. PubMed doi:10.1016/j.healthplace.2016.04.009

30. The American Academy of Pediatrics Council on Communications Media. Policy statement-children, adolescents, obesity, and the media. Pediatrics. 2011;128(1):201-208. PubMed doi:10.1542/ peds.2011-1066

31. Department of Education and Science. Primary school curriculum, physical education. Dublin 1999. Available from http://www.curriculumonline.ie/Primary/Curriculum. Accessed March 10, 2016.

32. Department of Education and Science. Junior cycle physical education. Dublin 1999. Available from https://curriculumonline.ie/getmedia/ ca078585-b4e2-4146-9d22-9fada4bd2478/JCSEC21_Physical_Edcuation_syllabus.pdf. Accessed March 10, 2016.

33. Department of Education Northern Ireland. Education curriculum minimum content. Bangor: Department of Education; 2007. Available from http://www.legislation.gov.uk/nisr/2007/46/pdfs/ nisr_20070046_en.pdf. Accessed March 10, 2016.

34. Callaghan M, Kelly C, Molcho M, Nic Gabhainn S. School characteristics, policy and context in Ireland: HBSC Ireland Post-Primary Schools. Galway, Ireland. Galway: Health Promotion Research Centre, National University of Ireland; 2013. Available from http:// www.nuigalway.ie/media/healthpromotionresearchcentre/hbscdocs/ shortreports/2015-Callaghan-School-characteristics.pdf. Accessed February 25, 2016.

35. Department of Education Statistics and Research Team NI. School omnibus survey 2014. Department of Education Northern Ireland; 2015. Available from https://www.education-ni.gov.uk/publications/ school-omnibus-survey-findings-2014. Accessed March 2, 2016.

36. Maitland C, Stratton G, Foster S, Braham R, Rosenberg M. A place for play? The influence of the home physical environment on children's physical activity and sedentary behaviour. Int J Behav Nutr Phys Act. 2013;10(1):99. PubMed doi:10.1186/1479-5868-10-99

37. Bauman AE, Reis RS, Sallis JF, Wells JC, Loos RJF, Martin BW. Correlates of physical activity: why are some people physically active and others not? Lancet. 2012;380(9838):258-271. PubMed doi:10.1016/ S0140-6736(12)60735-1

38. Department of Culture Arts and Leisure. Sport matters: Northern Ireland strategy for sport and physical recreation, 2009-19. Belfast: Sport Northern Ireland; 2009. Available from http://www.sportni.net/ about-us/sports-strategy/. Accessed January 28, 2016.

39. O'Keeffe B, O'Beirne A. Children's independent mobility on the island of Ireland. University of Limerick, Ireland 2015. Available from http:// www.mic.ul.ie/childmobility/Documents/Child\%20Mobility\%20 Report.pdf.

40. Sallis JF, Bull F, Guthold R, Heath GW, Inoue S, Kelly P, et al. Progress in physical activity over the Olympic quadrennium. Lancet. 2016:388(10051):1325-1336. doi:10.1016/S0140-6736(16)30581-5

41. Department of Health Social Services and Public Safety. A fitter future for all: Reviewed outcome framework 2015 - 2019. 2015. Available from https://www.health-ni.gov.uk/sites/default/files/publications/ dhssps/fitter-future-for-all-outcomes-framework-2015-2019.pdf. Accessed March 10, 2016. 